\title{
Magnetic Monopoles in the Deconfined Phase of Yang-Mills Theories
}

\section{Massimo D'Elia*}

Dipartimento di Fisica and INFN, Sezione di Genova, Via Dodecaneso 33, I-16146 Genova, ITALY

E-mail: delia@ge.infn.it

\section{Alessio D'Alessandro}

Dipartimento di Fisica and INFN, Sezione di Genova, Via Dodecaneso 33, I-16146 Genova, ITALY

E-mail: adales@ge.infn.it

We review recent lattice results, concerning the properties and the role of thermal abelian magnetic monopoles in the high temperature phase of Yang-Mills theories, and discuss preliminary evidence for the onset of their condensation as the deconfining temperature is approached from above.

International Workshop on QCD Green's Functions, Confinement, and Phenomenology - QCD-TNT09 September 07 - 112009

ECT Trento, Italy

${ }^{*}$ Speaker. 


\section{Introduction}

In the dual superconductor model [1,2], color confinement is related to the spontaneous breaking of a magnetic Abelian symmetry, induced by the condensation of Abelian magnetic monopoles. The magnetic condensate in the confined phase, as well as its disappearance at the deconfining transition, has been extensively investigated on the lattice $[3,4,5,6,7,8]$. Magnetically charged particles have also been supposed to be relevant to some physical properties of the Quark-Gluon Plasma phase above the transition $[9,10,11]$, such as its very low viscosity and diffusion coefficient and its strongly interacting, liquid-like nature.

In Ref. [10] the magnetic component of the Quark-Gluon Plasma (QGP) has been identified with Abelian magnetic monopoles evaporating from the condensate and becoming thermal particles for $T>T_{c}$. A definition was given for their detection on the lattice in terms of non-trivially wrapped trajectories in the Euclidean time direction (thermal monopoles). First numerical investigations of these wrapping trajectories were performed in Refs. $[12,13]$.

In Refs. [14, 15] we have applied the definition of Ref. [10] to find the physical properties of thermal monopoles in a wide range of temperatures above $T_{c}$. Simulations were done for $S U(2)$ pure gauge theory and various lattice sizes and lattice spacings. Monopole currents were identified by the usual De-Grand Toussaint construction [16], after Abelian projection in the Maximal Abelian Gauge (MAG).

Despite the dependence of the very definition of abelian monopoles on the abelian projection chosen, the density and the spatial correlation function of MAG thermal monopoles, which are shown respectively in Figs. 1 and 2, show a negligible dependence on the UV cut-off, as expected for a physical quantity. The temperature dependence of the monopole density, $\rho$, is not compatible with a (massive or massless) free particle behaviour in the whole range of temperatures explored, and is instead well described by a behaviour $\rho \propto T^{3} /\left(\log T / \Lambda_{\text {eff }}\right)^{2}$ with $\Lambda_{e f f} \sim 100 \mathrm{MeV}$, while the behaviour $\rho \propto T^{3} /(\log T)^{3}$, predicted by dimensional reduction arguments, is compatible with data for $T>5 T_{c}$. This is in agreement with the picture of an electric dominated phase for Yang-Mills theories at very high temperatures, in which the magnetic component is strongly interacting [9].

Moreover the study of density-density spatial correlation functions has verified the presence of a repulsive (attractive) interaction for a monopole-monopole (monopole-antimonopole) pair, which at large distances is in agreement with a screened Coulomb potential of screening length of the order of $0.1 \mathrm{fm}$. The above results have suggested a liquid-like behaviour for the thermal monopole ensemble above $T_{c}$ [17] and stimulated further research about the possible role of magnetic monopoles in the Quark-Gluon Plasma [18, 19].

In the present study we address a question regarding color confinement: if thermal monopoles in the deconfined phase are related to the magnetic condensate responsible for confinement at $T<T_{c}$, is it possible to find clear signatures for their approach to condensation for temperatures slightly above $T_{c}$ ? Since condensation is a phenomenon strictly related to the identity of quantum particles, one should look for the properties of thermal abelian monopole trajectories which reflect their quantum nature: as discussed in the following, such properties are encoded in monopole trajectories which wrap two or more times in the Euclidean time direction and correspond to two or more particles exchange. 


\section{Bose-Einstein condensation and monopole condensation}

In the path integral describing $N$ identical particles at thermal equilibrium, each possible configuration of the $N$ particle paths contributing to the functional integral needs to be periodic apart from a permutation of the $N$ particles; odd permutations give a negative contribution if the particles are fermions. Therefore in general each configuration is made up of $M$ closed paths, with $M \leq N$ : if the $j$-th path wraps $k_{j}$ times around the Euclidean time direction then $\sum_{j=1}^{M} k_{j}=N$ and the configuration corresponds to a permutation made up of $M$ cicles of sizes $k_{1}, k_{2}, \ldots k_{M}$. The case $M=N$ corresponds to the identical permutation. In the Boltzmann approximation configurations with $M<N$ have a negligible weight in the path integral, so that trajectories wrapping more than one time, corresponding to the exchange of two or more particles, are very rare. The number of trajectories wrapping more and more times is instead expected to increase as quantum effects become more important, as expected close to a transition induced by Bose-Einstein condensation (BEC).

For instance, a path integral formulation of the thermal partition function of an ideal nonrelativistic boson gas leads to following expression for the densities of paths wrapping $k$ times in the Euclidean time direction:

$$
\rho_{k} \equiv \frac{\left\langle n_{k}\right\rangle}{V}=\frac{e^{-\hat{\mu} k}}{\lambda^{3} k^{5 / 2}}
$$

where $n_{k}$ is the number of $k$-wrapping trajectories, $V$ is the available volume, $\lambda=\sqrt{2 \pi /(m T)}$

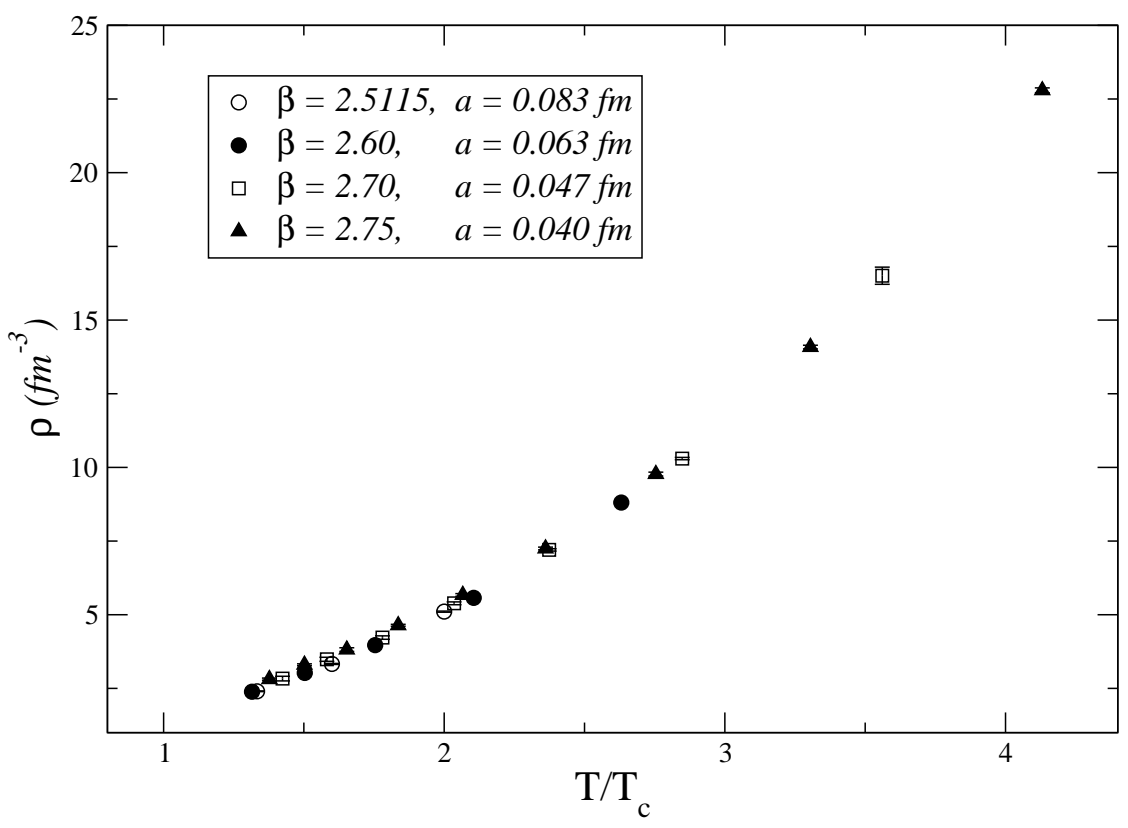

Figure 1: Monopole density, obtained after MAG gauge fixing, as a function of temperature, for different values of the lattice spacing $a(\beta)$. 


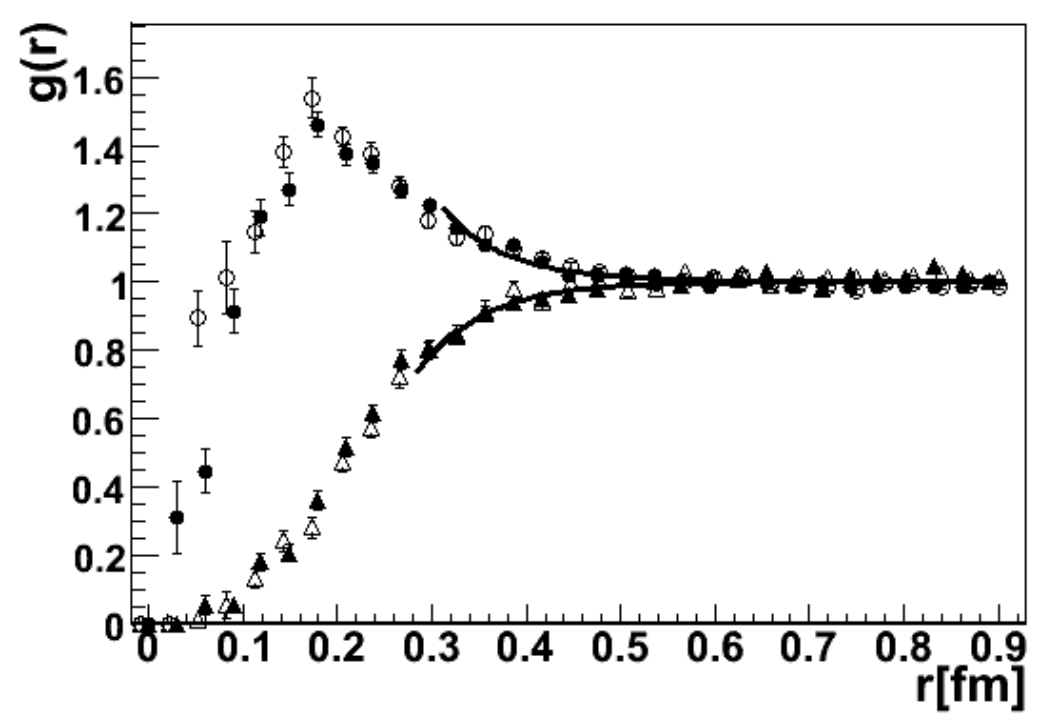

Figure 2: Monopole-antimonopole (circles) and monopole-monopole (triangles) spatial correlation function, for an inverse gauge coupling $\beta=2.70$ (empty markers) and $\beta=2.86$ (full markers).

is the de Broglie thermal wavelength and we have defined the dimensionless chemical potential $\hat{\mu} \equiv-\mu / T$, which is constrained to be non-negative (i.e. $\mu \leq 0$ ). The expression for the total particle density is then

$$
\rho=\sum_{k=1}^{\infty} k \rho_{k}=\frac{1}{\lambda^{3}} \sum_{k=1}^{\infty} \frac{e^{-\hat{\mu} k}}{k^{3 / 2}}=\frac{2}{\lambda^{3} \sqrt{\pi}} \int_{0}^{\infty} d x \frac{\sqrt{x}}{e^{\hat{\mu}} e^{x}-1}
$$

where in the last expression we have made use of the integral expression for the polylogarithm, thus recovering the usual expression for $\rho$ obtained when treating the free boson gas in momentum space. The total density is bounded by an upper limit which is reached for $\hat{\mu}=0$,

$$
\rho_{\max }=\frac{1}{\lambda^{3}} \sum_{k=1}^{\infty} \frac{1}{k^{3 / 2}} \simeq \frac{2.612}{\lambda^{3}}
$$

and after which Bose condensation begins. The approach to $\hat{\mu}=0$ is signalled, in the path integral approach, by a proliferation of trajectories with higher and higher wrapping number $k$, which are instead suppressed exponentially for $\hat{\mu} \neq 0$, see Eq. (2.1). The path integral approach to free boson condensation has been often taken as the starting point to describe the critical behaviour of more complex systems like liquid Helium [20, 21, 22, 23], and has been recently reconsidered in Ref. [24], where it has been suggested to apply it also to the analysis of monopole condensation in QCD.

The advantage of treating condensation at a path integral level is clear in view of the study of monopoles condensation: we have a direct connection with the information about the thermal monopole ensemble that can be retrieved by lattice QCD simulations. If we had a set of trajectories sampled from the path integral of an ideal non-relativistic bosonic gas at various different 


\begin{tabular}{|c|c|c|c|c|c|c|}
\hline$T / T_{c}$ & $\rho_{1} / T^{3}$ & $\rho_{2} / T^{3}$ & $\rho_{3} / T^{3}$ & $\rho_{4} / T^{3}$ & $\rho_{5} / T^{3}$ & $\rho_{6} / T^{3}$ \\
\hline $1.017^{a}$ & $0.308(2)$ & $1.53(1) 10^{-2}$ & $3.40(5) 10^{-3}$ & $1.21(3) 10^{-3}$ & $4.1(3) 10^{-4}$ & $2.0(3) 10^{-4}$ \\
\hline $1.052^{b}$ & $0.315(5)$ & $1.35(1) 10^{-2}$ & $2.42(4) 10^{-3}$ & $7.0(2) 10^{-4}$ & $2.5(2) 10^{-4}$ & $8.2(5) 10^{-5}$ \\
\hline $1.095^{a}$ & $0.3395(15)$ & $1.23(2) 10^{-2}$ & $1.81(5) 10^{-3}$ & $4.1(4) 10^{-4}$ & $0.9(1) 10^{-4}$ & $2.3(5) 10^{-5}$ \\
\hline $1.168^{b}$ & $0.325(3)$ & $8.0(1) 10^{-3}$ & $7.6(2) 10^{-4}$ & $1.2(1) 10^{-4}$ & $1.1(3) 10^{-5}$ & $0.2(1) 10^{-5}$ \\
\hline $1.187^{a}$ & $0.337(2)$ & $8.1(1) 10^{-3}$ & $7.3(4) 10^{-4}$ & $1.1(1) 10^{-4}$ & $1.5(4) 10^{-5}$ & $0.4(2) 10^{-5}$ \\
\hline $1.295^{a}$ & $0.316(1)$ & $4.72(10) 10^{-3}$ & $2.6(3) 10^{-4}$ & $2.0(6) 10^{-5}$ & $0.3(2) 10^{-5}$ & \\
\hline $1.315^{b}$ & $0.297(2)$ & $3.83(3) 10^{-3}$ & $1.7(1) 10^{-4}$ & $1.3(2) 10^{-5}$ & $0.6(3) 10^{-6}$ & \\
\hline $1.424^{a}$ & $0.286(1)$ & $2.52(5) 10^{-3}$ & $8.4(7) 10^{-5}$ & $0.4(2) 10^{-5}$ & & \\
\hline $1.503^{b}$ & $0.271(1)$ & $1.78(5) 10^{-3}$ & $4.1(3) 10^{-5}$ & $0.8(4) 10^{-6}$ & & \\
\hline $1.582^{a}$ & $0.252(1)$ & $1.28(2) 10^{-3}$ & $2.5(3) 10^{-5}$ & $1.0(5) 10^{-6}$ & & \\
\hline $1.754^{b}$ & $0.2134(10)$ & $7.3(3) 10^{-4}$ & $9(1) 10^{-6}$ & $0.2(1) 10^{-6}$ & & \\
\hline $1.780^{a}$ & $0.2190(2)$ & $6.26(7) 10^{-4}$ & $8.3(8) 10^{-6}$ & $0.2(1) 10^{-6}$ & & \\
\hline $2.034^{a}$ & $0.1870(4)$ & $3.04(10) 10^{-4}$ & $1.0(4) 10^{-6}$ & & & \\
\hline
\end{tabular}

Table 1: Normalized densities $\rho_{k} / T^{3}$ as a function of $T / T_{c}$. The superscript $a$ or $b$ above the temperature value refers to two different values of the inverse gauge coupling, $\beta=2.70$ and $\beta=2.60$.

temperatures, we could measure the distribution of $k$-cycles, i.e. the densities $\rho_{k}$, and then by fitting the expected dependence given in Eq. (2.1) we could determine the chemical potential $\hat{\mu}$ as a function of $T$ in order to find numerically the critical temperature $T_{\mathrm{BEC}}$ at which $\hat{\mu}$ vanishes and Bose-Einstein condensation begins. Our aim is to repeat a similar analysis starting from the ensemble of thermal monopole trajectories.

\section{Numerical Results}

In Table 2 we report the normalized average density $\rho_{k} / T^{3}$ of trajectories wrapping $k$ times as a function of temperature. Data include both monopole and antimonopole trajectories and have been obtained by extracting monopole currents from samples consisting of $O\left(10^{3}\right)$ independent gauge configurations for each value of $T$ and at two different values of the inverse gauge coupling, $\beta=2.70$ and $\beta=2.60$, corresponding respectively to lattice spacings $a \simeq 0.047 \mathrm{fm}$ and $a \simeq 0.063$ $\mathrm{fm}$. The physical scale has been determined according to $a(\beta) \Lambda_{L}=R(\beta) \lambda(\beta)$, where $R$ is the two-loop perturbative $\beta$-function, while $\lambda$ is a non-perturbative correction factor computed and reported in Ref. [25]. We have assumed the values $T_{c} / \Lambda_{L}=21.45$ (14) [25], $T_{c} / \sqrt{\sigma}=0.69$ (2) [26] and $\sqrt{\sigma} \simeq 430 \mathrm{MeV}$.

Data obtained for each $\rho_{k}$ show a good independence from the value of the UV cutoff. The relative weight of trajectories wrapping more than one time grows rapidly as $T$ approaches $T_{c}$. The number of monopoles or antimonopoles which are found in non-trivial cycles (i.e. $k>1$ ) is less than $0.1 \%$ for $T>2.5 T_{c}$, meaning that the system is essentially Boltzmann-like at high temperatures. The same number goes to about $1 \%$ for $T \sim 1.5 T_{c}$ and is well above $10 \%$ at the lowest temperature explored in our simulations. This is a clear qualitative signature for quantum 


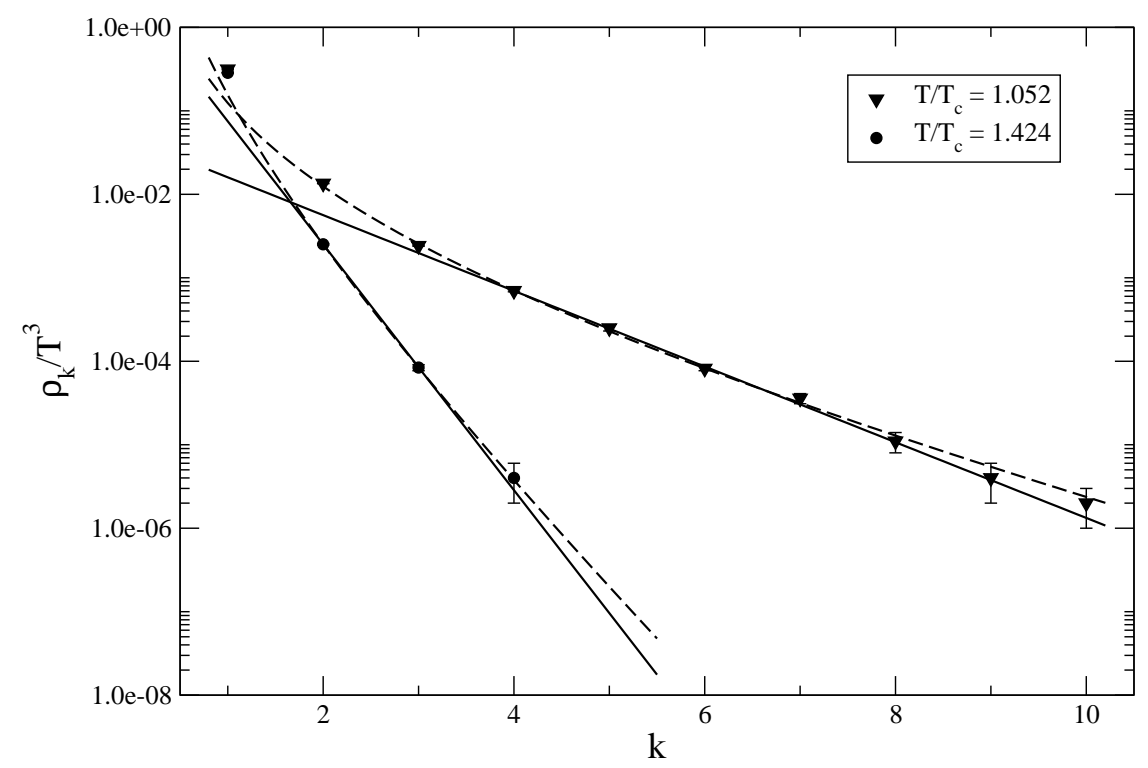

Figure 3: Fit of the densities $\rho_{k}$ according to $e^{-\hat{\mu} k} / k^{5 / 2}$ (dashed line) and according to $e^{-\hat{\mu} k}$ (solid line) for two values of the temperature.

statistics effects becoming more and more relevant as the critical temperature is approached, as expected if monopoles condense at $T_{c}$.

In order to make a quantitative analysis, we need to make an assumption about the dependence of $\rho_{k}$ on $k$. The ensemble of thermal abelian monopoles that we are investigating is surely different from an ideal gas of non-relativistic bosons. The analysis of Ref. [14] indeed has shown the presence of particle-particle interactions, which are attractive in the monopole-antimonopole case and repulsive in the monopole-monopole case. As a first approximation, however, we can check if a dependence similar to that expected for free bosons describes well our data. In particular, we have tried the following ansatz

$$
\rho_{k}=e^{-\hat{\mu} k} / k^{\alpha}
$$

and verified that reasonable fits are obtained for various different values of $\alpha$ ranging from 0 to 3; some examples are reported in Fig. 3.

Of course the values of the chemical potentials obtained in this way depend on the value used for $\alpha$ : this is apparent from the results reported in Fig. 4. It is however remarkable that one can try to fit the values obtained for the chemical potentials according to a critical behaviour:

$$
\hat{\mu}=A\left(T-T_{\mathrm{BEC}}\right)^{v^{\prime}}
$$

obtaining as a result that $\hat{\mu}$ vanishes at a condensation temperature $T_{\mathrm{BEC}}$ which, independently of the value of $\alpha$, coincides within errors with the deconfinement temperature $T_{c}$. This is apparent from Fig. 4 in which we have reported results obtained for two different values of $\alpha$ and descarding the lowest temperature, $T=1.017 T_{c}$, from the fits. 


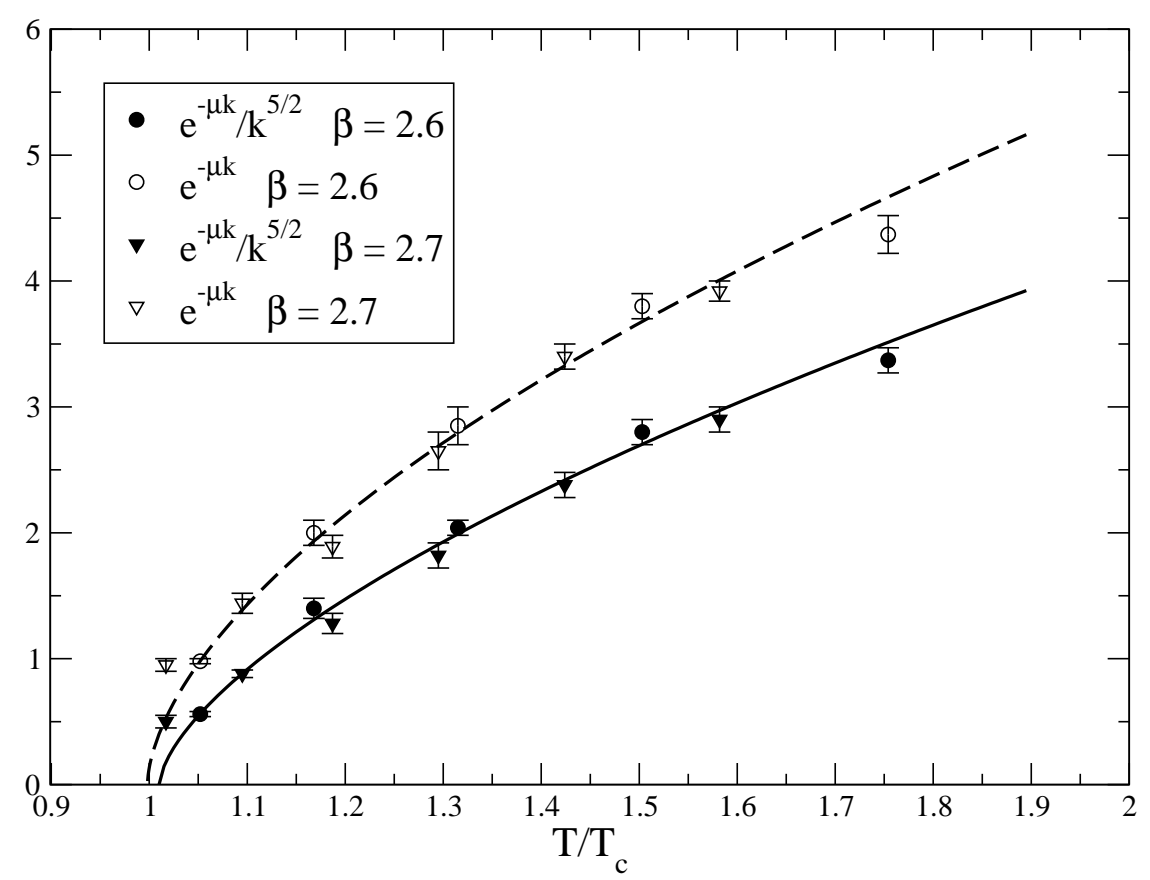

Figure 4: Chemical potentials obtained for $\alpha=0$ and $\alpha=5 / 2$ (see Eq. (3.1)) together with fits according to Eq. (3.2).

\section{Conclusions}

The possible role of magnetic degrees of freedom in the dynamics of strongly interacting nonabelian gauge theories has been emphasized since long. They are believed to play a fundamental role in the mechanism of Color Confinement. Moreover it has been claimed recently that magnetic degrees of freedom may explain many properties of strongly interacting matter in the high temperature, deconfined phase.

In Ref. [14] we have performed a first extensive investigation about the physical properties of thermal magnetic degrees of freedom, identified after abelian projection according to a proposal given in Ref. [10], in the deconfined phase of $S U$ (2) pure gauge theory. In the present study we have provided preliminary evidence that, as we proceed from higher to lower temperatures, the onset of confinement is associated with the condensation of thermal monopoles present in the deconfined phase, as expected in the dual superconductor scenario for color confinement.

Further studies should put this result on a firmer basis, by taking properly into account the dynamics of monopole interactions above $T_{c}$, as well as by extending the investigation to three colors and including dynamical quark contributions.

\section{References}

[1] G. 't Hooft, in “High Energy Physics”, EPS International Conference, Palermo 1975, ed. A. Zichichi. 
[2] S. Mandelstam, Phys. Rept. 23, 245 (1976).

[3] A. Di Giacomo, B. Lucini, L. Montesi, G. Paffuti, Colour confinement and dual superconductivity of the vacuum. I, Phys. Rev. D 61 (2000) 034503 [arXiv: hep-lat/9906024]; Colour confinement and dual superconductivity of the vacuum. II, Phys. Rev. D 61 (2000) 034504 [arXiv: hep-lat/9906025].

[4] J. M. Carmona, M. D’Elia, A. Di Giacomo, B. Lucini, G. Paffuti, Color confinement and dual superconductivity of the vacuum. III, Phys. Rev. D 64 (2001) 114507 [arXiv: hep-lat/0103005].

[5] J. M. Carmona, M. D’Elia, L. Del Debbio, A. Di Giacomo, B. Lucini, G. Paffuti, Color confinement and dual superconductivity in full QCD Phys. Rev. D 66 (2002) 011503 [arXiv: hep-lat/0205025].

[6] M. D’Elia, A. Di Giacomo, B. Lucini, G. Paffuti, C. Pica, Color confinement and dual superconductivity of the vacuum. IV, Phys. Rev. D 71 (2005) 114502 [arXiv: hep-lat/0503035].

[7] M.N. Chernodub, M.I. Polikarpov and A.I. Veselov, Phys. Lett. B399, 267 (1997).

[8] P. Cea and L. Cosmai, Abelian monopole and vortex condensation in lattice gauge theories, JHEP 0111 (2001) 064; P. Cea, L. Cosmai and M. D'Elia, The deconfining phase transition in full QCD with two dynamical flavors, JHEP 0402 (2004) 018.

[9] J. Liao and E. Shuryak, Strongly coupled plasma with electric and magnetic charges, Phys. Rev. C 75 (2007) 054907 [hep-ph/0611131].

[10] M. N. Chernodub and V. I. Zakharov, Magnetic component of Yang-Mills plasma, Phys. Rev. Lett. 98 (2007) 082002 [arXiv: hep-ph/0611228]; M. N. Chernodub and V. I. Zakharov, Magnetic strings as part of Yang-Mills plasma, arXiv: hep-ph/0702245.

[11] E. Shuryak, Physics of Strongly coupled Quark-Gluon Plasma, Prog. Part. Nucl. Phys. 62 (2009) 48 [arXiv:0807.3033 [hep-ph]].

[12] V.G. Bornyakov, V.K. Mitrjushkin and M. Muller-Preussker Phys. Lett. B284, 99 (1992).

[13] S. Ejiri, Monopoles and Spatial String Tension in the High Temperature Phase of SU(2) QCD, Phys. Lett. B376 (1996) 163 [arXiv: hep-lat/9510027].

[14] A. D'Alessandro and M. D'Elia, Magnetic monopoles in the high temperature phase of Yang-Mills theories, Nucl. Phys. B 799 (2008) 241.

[15] A. D'Alessandro and M. D'Elia, Magnetic monopoles in high temperature QCD, PoS CONFINEMENT8 (2008) 127 [arXiv: 0812.1867 [hep-lat]].

[16] A. De Grand and D. Toussaint, Topological excitations and Monte Carlo simulation of Abelian gauge theory, Phys. Rev. D 22 (1980) 2478.

[17] J. Liao and E. Shuryak, Magnetic Component of Quark-Gluon Plasma is also a Liquid!, Phys. Rev. Lett. 101 (2008) 162302 [arXiv: 0804.0255 [hep-ph]].

[18] C. Ratti and E. Shuryak, The role of monopoles in a Gluon Plasma, Phys. Rev. D 80 (2009) 034004.

[19] M. N. Chernodub, A. D'Alessandro, M. D’Elia and V. I. Zakharov, Thermal monopoles and selfdual dyons in the Quark-Gluon Plasma, [arXiv:0909.5441 [hep-lat]].

[20] R.P. Feynman, The ג-Transition in Liquid Helium, Phys. Rev. 90 (1953) 1116. 
[21] R.P. Feynman, Atomic Theory of the lamda Transition in Helium, Phys. Rev. 91 (1953) 1291.

[22] V. Elser, Topics in Statistical Mechanics, Ph.D. Thesis, U.C. Berkeley, (1984).

[23] D.M. Ceperley, Path integrals in the theory of condensed Helium, Rev. Mod. Phys. 67 (1995) 279.

[24] M. Cristoforetti and E. Shuryak, Bose-Einstein Condensation of strongly interacting bosons: from liquid ${ }^{4}$ He to QCD monopoles, Phys. Rev. D 80 (2009) 054013.

[25] J. Engels, F. Karsch, K. Redlich, Scaling properties of the energy density in SU(2) lattice gauge theory, Nucl. Phys. B 435 (1995) 295 [arXiv: hep-lat/ 9408009 ].

[26] J. Fingberg, U. Heller, F. Karsch, Scaling and asymptotic scaling in the SU(2) gauge theory, Nucl. Phys. B 392 (1992) 493 [arXiv: hep-lat/9208012]. 